\title{
Solvability of non-semicontinuous systems of Stieltjes differential inclusions and equations
}

\author{
Rodrigo López Pouso ${ }^{1}$, Ignacio Márquez Albés ${ }^{1 *}$ (iD and Jorge Rodríguez-López ${ }^{1}$
}

\section{"Correspondence:}

ignacio.marquez@usc.es

'Departamento de Estatística,

Análise Matemática e Optimización

Instituto de Matemáticas, Facultade de Matemáticas, Universidade de

Santiago de Compostela, Santiago, Spain

\begin{abstract}
We prove an existence result for systems of differential inclusions driven by multivalued mappings which need not assume closed or convex values everywhere, and need not be semicontinuous everywhere. Moreover, we consider differentiation with respect to a nondecreasing function, thus covering discrete, continuous and impulsive problems under a unique formulation. We emphasize that our existence result appears to be new even when the derivator is the identity, i.e. when derivatives are considered in the usual sense. We also apply our existence theorem for inclusions to derive a new existence result for discontinuous Stieltjes differential equations. Examples are given to illustrate the main results.
\end{abstract}

MSC: $34 \mathrm{~A} 60 ; 34 \mathrm{~A} 36$

Keywords: Differential inclusions; Discontinuous differential equations; Stieltjes differential inclusions; Stieltjes differential equations

\section{Introduction}

Stieltjes differential equations are equations of the form

$$
x_{g}^{\prime}(t)=f(t, x(t)), \quad t \in\left[t_{0}, t_{0}+T\right),\left(t_{0} \in \mathbb{R}, T>0\right),
$$

where, roughly speaking, the Stieltjes derivative of $x$ with respect to $g$ is

$$
x_{g}^{\prime}(t)=\lim _{s \rightarrow t} \frac{x(s)-x(t)}{g(s)-g(t)}
$$

where the derivator function $g: \mathbb{R} \longrightarrow \mathbb{R}$ is nondecreasing. We include in Sect. 2 the precise definitions and basic properties established in $[7,8]$.

The definition of Stieltjes derivative is consistent with Stieltjes integration, in the sense that every bonna fide (absolutely continuous) function can be recovered as the indefinite Lebesgue-Stieltjes integral of its $g$-derivative. Although we shall constantly refer to results in [8], we remark that there exist much older, similar notions of derivatives with respect to functions and fundamental theorems for Stieltjes integrals, see for example [3].

(c) The Author(s) 2020. This article is licensed under a Creative Commons Attribution 4.0 International License, which permits use sharing, adaptation, distribution and reproduction in any medium or format, as long as you give appropriate credit to the original author(s) and the source, provide a link to the Creative Commons licence, and indicate if changes were made. The images or other third party material in this article are included in the article's Creative Commons licence, unless indicated otherwise in a credit line to the material. If material is not included in the article's Creative Commons licence and your intended use is not permitted by statutory regulation or exceeds the permitted use, you will need to obtain permission directly from the copyright holder. To view a copy of this licence, visit http://creativecommons.org/licenses/by/4.0/. 
As explained in [8], the importance of Stieltjes derivatives is that (1.1) offers a technically simple unified framework for difference and differential equations, and even allows for impulses on countable sets of fixed times. In a similar way, it is shown in [9] that Stieltjes differential inclusions represent a more general context for the study of impulsive differential inclusions. In Sect. 3 we focus our attention to Stieltjes differential inclusions of the form

$$
x_{g}^{\prime}(t) \in F(t, x(t)), \quad t \in\left[t_{0}, t_{0}+T\right),
$$

and we obtain an existence result which, to the best of our knowledge, is new even in the particular case when $g$ is the identity, i.e. the case of usual derivatives-thus generalizing the results in [2]. Finally, in Sect. 4 we use the result we obtained for (1.2) to obtain a new existence result for (1.1) with discontinuous functions $f(t, x)$.

\section{Preliminares}

Let $g: \mathbb{R} \rightarrow \mathbb{R}$ be a nondecreasing left-continuous function. Let us recall the definition of the $g$-derivative introduced in [8]. To that end, we first introduce the following two sets: first, the set of points around which $g$ is constant,

$$
C_{g}=\{t \in \mathbb{R}: g \text { is constant on }(t-\varepsilon, t+\varepsilon) \text { for some } \varepsilon>0\} \text {, }
$$

and, second, the set of discontinuity points of $g$, which can be expressed as

$$
D_{g}=\left\{t \in \mathbb{R}: g\left(t^{+}\right)-g(t)>0\right\},
$$

where $g\left(t^{+}\right)$denotes the limit of $g$ at $t$ from the right.

Now the $g$-derivative of a real valued function $x=x(t)$ at a point $t \in \mathbb{R} \backslash C_{g}$ is

$$
x_{g}^{\prime}(t)= \begin{cases}\lim _{s \rightarrow t} \frac{x(s)-x(t)}{g(s)-g(t)}, & \text { if } t \notin D_{g}, \\ \frac{x\left(t^{+}\right)-x(t)}{g\left(t^{+}\right)-g(t)}, & \text { if } t \in D_{g},\end{cases}
$$

provided that the corresponding limit exists.

Notice that we do not define $g$-derivatives at points $t \in C_{g}$, nor is it necessary because $C_{g}$ is a null-measure set for $\mu_{g}$ (the Lebesgue-Stieltjes measure induced by $g$ ); see [8, Proposition 2.5]. Notice also that

$$
\mu_{g}(\{t\})=g\left(t^{+}\right)-g(t)=0 \quad \text { if and only if } \quad t \in \mathbb{R} \backslash D_{g} .
$$

In this paper we shall consider integration in the Lebesgue-Stieltjes sense, and we shall call " $g$-measurable" any function (or set) which is measurable with respect to the Lebesgue-Stieltjes $\sigma$-algebra generated by $g$.

The following concept of $g$-absolute continuity is crucial in this paper. We consider the maximum norm in $\mathbb{R}^{n}$. 
Definition 2.1 Let $a, b \in \mathbb{R}, a<b$. We say that a function $f:[a, b] \longrightarrow \mathbb{R}^{n}$ is absolutely continuous with respect to $g$ on $[a, b]$ (or $g$-absolutely continuous on $[a, b]$, and we denote it by $\left.f \in \mathcal{A C}_{g}([a, b])\right)$ if to each $\varepsilon>0$ there is some $\delta>0$ such that, for any family $\left\{\left(a_{n}, b_{n}\right)\right\}_{n=1}^{m}$ of pairwise disjoint open subintervals of $[a, b]$, the inequality

$$
\sum_{n=1}^{m}\left(g\left(b_{n}\right)-g\left(a_{n}\right)\right)<\delta
$$

implies

$$
\sum_{n=1}^{m}\left\|f\left(b_{n}\right)-f\left(a_{n}\right)\right\|<\varepsilon
$$

Next we state the two versions of the fundamental theorem of calculus.

Theorem 2.2 ([8, Theorem 2.4, Proposition 5.2]) Let $a, b \in \mathbb{R}, a<b$, and $c:[a, b) \longrightarrow \mathbb{R}^{n}$. Assume that $c \in L_{g}^{1}([a, b))$, the Banach space of Lebesgue-Stieltjes integrable functions with respect to $\mu_{g}$, and consider its indefinite Lebesgue-Stieltjes integral

$$
C(t)=\int_{[a, t)} c(s) d g(s) \quad \text { for all } t \in[a, b]
$$

Then $C$ is $g$-absolutely continuous on $[a, b]$ and there is a $g$-measurable set $N \subset[a, b]$ such that $\mu_{g}(N)=0$ and

$$
C_{g}^{\prime}(t)=c(t) \quad \text { for all } t \in[a, b) \backslash N \text {. }
$$

Theorem 2.3 (Fundamental theorem of calculus for the Lebesgue-Stieltjes integral) Let $a, b \in \mathbb{R}, a<b$, and $f:[a, b] \longrightarrow \mathbb{R}^{n}$. The following conditions are equivalent.

(1) The function $f$ is $g$-absolutely continuous on $[a, b]$.

(2) The function $f$ fulfills the following properties:

(a) There exists $f_{g}^{\prime}(t)$ for $g$-almost all $t \in[a, b)$ (i.e., for all $t \in[a, b)$ except on a set of $\mu_{g}$-measure zero).

(b) $f_{g}^{\prime} \in L_{g}^{1}([a, b))$.

(c) For each $t \in[a, b]$, we have

$$
f(t)=f(a)+\int_{[a, t)} f_{g}^{\prime}(s) d g(s) .
$$

Remark 2.4 Observe that $f_{g}^{\prime}(b)$ plays no role in Theorem 2.3. This is natural because $\mu_{g}(\{b\})=0$ if $b \notin D_{g}$ (so we do not have to worry about $f_{g}^{\prime}(b)$ ), and if $b \in D_{g}$ then $f_{g}^{\prime}(b)$ does not exist because $f(t)$ is not defined for $t>b$.

For more properties of $g$-absolutely continuous functions we refer to [7, 8]. For selfcontainedness, we include the following result.

Proposition 2.5 ([8, Proposition 5.3]) Iff is $g$-absolutely continuous on $[a, b]$, then it has bounded variation and it is continuous from the left at every $t \in[a, b)$. Moreover, $f$ is con- 
tinuous in $[a, b] \backslash D_{g}$, and if $g$ is constant on some interval $(\alpha, \beta) \subset[a, b]$, then $f$ is constant on $(\alpha, \beta)$ as well.

Finally, we recall the concept of $g$-continuity and its relation with $g$-absolute continuity.

Definition 2.6 Let $f:[a, b] \longrightarrow \mathbb{R}^{n}$ and $t \in[a, b]$. We say that $f$ is $g$-continuous at $t$ if for every $\varepsilon>0$ there exists $\delta>0$ such that

$$
[s \in[a, b],|g(s)-g(t)|<\delta] \Rightarrow\|f(s)-f(t)\|<\varepsilon .
$$

We say that $f$ is $g$-continuous on $[a, b]$ if it is $g$-continuous at every point $t \in[a, b]$.

We denote by $\mathcal{B C}_{g}([a, b])$ the set of $g$-continuous bounded functions. It is proven in [7] that $\mathcal{B C}_{g}([a, b])$ is a Banach space with the supremum norm and, moreover, $\mathcal{A C}_{g}([a, b]) \subset$ $\mathcal{B C}_{g}([a, b])$. We have the following sufficient condition for relative compactness of sets of $g$-absolutely continuous functions in $\mathcal{B C}_{g}([a, b])$

Proposition 2.7 ([7, Proposition 5.6]) Let $\mathcal{S} \subset \mathcal{A C}_{g}([a, b])$ be such that $\{f(a): f \in \mathcal{S}\}$ is bounded. Assume that there exists $h \in L_{g}^{1}([a, b))$ such that

$$
\left\|f_{g}^{\prime}(t)\right\| \leq h(t) \quad \text { for } g \text {-almost all } t \in[a, b) \text {, and for all } f \in \mathcal{S} \text {. }
$$

Then $\mathcal{S}$ is relatively compact in $\mathcal{B C}_{g}([a, b])$.

\section{Stieltjes differential inclusions}

Let $t_{0}, T \in \mathbb{R}, T>0$, and consider the intervals

$$
I=\left[t_{0}, t_{0}+T\right] \text { and } J=\left[t_{0}, t_{0}+T\right) \text {. }
$$

In this section we study the existence of $\mathcal{A C}_{g}$-solutions of the problem

$$
x_{g}^{\prime}(t) \in F(t, x(t)) \quad \text { for } g \text {-a.a. } t \in J, \quad x\left(t_{0}\right)=x_{0},
$$

where $x_{0} \in \mathbb{R}^{n}$ and $F: J \times \mathbb{R}^{n} \rightarrow \mathcal{P}\left(\mathbb{R}^{n}\right)$ are given, where $\mathcal{P}\left(\mathbb{R}^{n}\right)$ denotes the set of all subsets of $\mathbb{R}^{n}$. Every multivalued mapping considered in this paper shall be assumed to be strict, i.e., to assume nonempty values.

Definition 3.1 A solution of (3.2) is a function $x \in \mathcal{A C}_{g}(I)$ such that $x\left(t_{0}\right)=x_{0}$ and

$$
x_{g}^{\prime}(t) \in F(t, x(t)), \quad g \text {-a.a. } t \in J .
$$

We shall prove an existence result for problem (3.2) by generalizing the ideas of [4], who studied the particular case when $g$ is the identity and assumed upper semicontinuity of $F(t, \cdot)$ on $\mathbb{R}^{n}$ for a.a. $t \in I$. In this paper, besides considering derivatives in a wider sense, we show that upper semicontinuity may fail at many points provided that they belong to the graph of another multivalued mapping satisfying some technical conditions with respect to $F$. 
We shall base our arguments on the following new concept of contingent $g$-derivative for multivalued mappings. This concept is based on the analytical description of the contingent derivative in the usual sense given by [1, Proposition 2, p. 177].

Definition 3.2 Let $K: I_{K} \rightarrow \mathcal{P}\left(\mathbb{R}^{n}\right)$ where $I_{K}$ is a nonempty subset of the reals.

The contingent $g$-derivative of $K$ at a point $(t, x) \in \operatorname{graph}(K)$ is the set denoted by $\mathrm{D}_{g} K(t, x)$ defined as follows: we say that $v \in \mathrm{D}_{g} K(t, x)$ if there exist $\left\{h_{k}\right\}_{k \in \mathbb{N}} \subset \mathbb{R}, t+h_{k} \in I_{K}$ for $k \in \mathbb{N}$, and $\left\{x_{k}\right\}_{k \in \mathbb{N}} \subset \mathbb{R}^{n}$ such that

1. $h_{k}>0$ for all $k \in \mathbb{N}$ and $\left\{h_{k}\right\}_{k \in \mathbb{N}}$ converges to 0 ;

2. $x_{k} \in K\left(t+h_{k}\right)$ for all $k \in \mathbb{N}$ and

$$
\lim _{k \rightarrow \infty} \frac{x_{k}-x}{g\left(t+h_{k}\right)-g(t)}=v
$$

Remark 3.3 In the conditions of Definition 3.2, if $t \notin D_{g}$, then $\left\{x_{k}\right\}_{k \in \mathbb{N}}$ necessarily converges to $x$. Indeed, in that case we see that $g\left(t+h_{k}\right)$ converges to $g(t)$ so

$$
0 \leq\left\|x_{k}-x\right\|=\left\|\frac{x_{k}-x}{g\left(t+h_{k}\right)-g(t)}\right\|\left(g\left(t+h_{k}\right)-g(t)\right) \stackrel{k \rightarrow \infty}{\longrightarrow} 0 .
$$

For a better understanding of this definition, let us consider $K(t)=\{\gamma(t)\}$ for some $\gamma$ : $I_{\gamma} \rightarrow \mathbb{R}^{n}$. Then it follows directly from the definition that, if $\gamma$ is $g$-differentiable from the right at a point $t_{0} \in I_{\gamma}$, then $\mathrm{D}_{g} K\left(t_{0}, \gamma\left(t_{0}\right)\right)=\left\{\gamma_{g}^{\prime}\left(t_{0}\right)\right\}$. Remember that to be $g$-differentiable from the right is equivalent to being $g$-differentiable for points in $D_{g}$.

Equipped with the notion of contingent $g$-derivative we can introduce our assumptions on $F(t, x)$. We assume that $F: J \times \mathbb{R}^{n} \rightarrow \mathcal{P}\left(\mathbb{R}^{n}\right)$ satisfies the following conditions, in the spirit of [2]:

(H1) (i) for all $t \in J \cap D_{g}, F(t, \cdot)$ assumes convex and compact values and it is upper semicontinuous on $\mathbb{R}^{n}$;

(ii) for $g$-a.a. $t \in J \backslash D_{g}, F(t, x)$ is convex and compact for every $x \in \mathbb{R}^{n} \backslash K(t)$, and $F(t, \cdot)$ is upper semicontinuous on $\mathbb{R}^{n} \backslash K(t)$, where the set $K(t)$ is either empty, or there exist $K_{p}: I_{p} \subset J \backslash D_{g} \longrightarrow \mathcal{P}\left(\mathbb{R}^{n}\right), p \in \mathbb{N}$, such that

$$
K(t)=\bigcup_{\left\{p: t \in I_{p}\right\}} K_{p}(t)
$$

and if $x \in K_{p}(t)$ for some $p$, then

$$
\bigcap_{r>0}^{\infty} \overline{\mathrm{co}} F\left(t, B_{r}(x)\right) \cap \mathrm{D}_{g} K_{p}(t, x) \subset F(t, x),
$$

where $\overline{c o}$ means closed convex hull and $B_{r}(x)$ is the open ball centered at $x$ with radius $r>0$;

(H2) for all $x \in \mathbb{R}^{n}$, there exists a $g$-measurable selection of $F(\cdot, x)$, that is, there exists a $g$-measurable function $f_{x}: J \rightarrow \mathbb{R}^{n}$ such that $f_{x}(t) \in F(t, x)$ for $g$-a.a. $t \in J$;

(H3) there exists $M \in L_{g}^{1}(J,[0, \infty))$ such that, for all $x \in \mathbb{R}^{n}$ and $g$-a.a. $t \in J$,

$$
\|y\| \leq M(t) \quad \text { for any } y \in F(t, x) .
$$


Remark 3.4 Note that $(\mathrm{H} 3)$ ensures that the $g$-measurable selections in $(\mathrm{H} 2)$ are $g$ integrable on $J$. Indeed, let $x \in \mathbb{R}^{n}$ be fixed and $f_{x}$ a selection in the conditions of (H2). Then $f_{x}(t) \in F(t, x)$ for $g$-a.a. $t \in J$, so (H3) implies that

$$
\int_{\left[t_{0}, t_{0}+T\right)}\left\|f_{x}(t)\right\| d g(t) \leq \int_{\left[t_{0}, t_{0}+T\right)} M(t) d g(t)<+\infty
$$

First, we recall the following result that characterizes weakly sequentially compact sets of integrable functions (see [6, Theorem IV.8.9]).

Theorem 3.5 Let $(X, \mathcal{M}, \mu)$ be a measure space and let $L_{\mu}^{1}(X)$ denote the set of $\mu$-integrable functions. A set $\mathcal{S} \subset L_{\mu}^{1}(X)$ is weakly sequentially compact if and only if

1. $\mathcal{S}$ is bounded;

2. for each $\left\{E_{m}\right\}_{m \in \mathbb{N}} \subset \mathcal{M}$ such that $E_{m+1} \subset E_{m}, m \in \mathbb{N}$, and $\bigcap_{m \in \mathbb{N}} E_{m}=\emptyset$, we have

$$
\lim _{m \rightarrow \infty} \int_{E_{m}} f d \mu=0 \quad \text { uniformly for } f \in \mathcal{S} .
$$

We also need [6, Corollary V.3.14], a result that ensures that it is possible to obtain a sequence converging strongly to a point in a Banach space from one that converges weakly to that same point.

Theorem 3.6 Let $X$ be a Banach space and $\left\{x_{m}\right\}_{m \in \mathbb{N}}$ a sequence in $X$ converging weakly to $x$. Then some sequence of convex combinations of the elements $x_{m}$ converges strongly to $x$.

Finally, we recall the following elementary topological property that will be key for the proof of the main result of this section.

Lemma 3.7 Let $S \subset \mathbb{R}^{n}$ be a compact set in the usual topology and let $x \in \mathbb{R}^{n} \backslash S$. Then there exists $\varepsilon_{0}>0$ such that $x \notin S+B_{\varepsilon_{0}}(0)$.

Proof The mapping $\varphi(y)=\|x-y\|$ is continuous on $\mathbb{R}^{n}$. Since $S$ is compact, $\varphi$ attains a minimum in $S$, i.e., there exists $y_{0} \in S$ such that $\|x-y\|=\varphi(y) \geq \varphi\left(y_{0}\right)$ for all $y \in S$ and $\varphi\left(y_{0}\right)>0$ because $x \neq y_{0}$. Therefore $x \notin S+B_{\varepsilon_{0}}(0)$ for $\varepsilon_{0}=\varphi\left(y_{0}\right)$.

We can now state and prove the following lemma that gives us some information about pointwise limits of sequences of $g$-absolutely continuous functions and their $g$-derivatives. This result can be seen as a generalization of [4, Theorem 4.1].

Lemma 3.8 Let I and $J$ be as in (3.1) and let $x_{k}: I \rightarrow \mathbb{R}^{n}, k \in \mathbb{N}$, be a sequence of functions such that

(i) $x_{k} \in \mathcal{A C}_{g}(I), k \in \mathbb{N}$;

(ii) $x_{k}(t) \rightarrow x(t)$ for all $t \in I$ for some $x: I \rightarrow \mathbb{R}^{n}$;

(iii) there exists $L \in L_{g}^{1}(J)$ such that for every $k \in \mathbb{N}$ we have

$$
\left\|\left(x_{k}\right)_{g}^{\prime}(t)\right\| \leq L(t), \quad \text { g-a.a. } t \in J
$$


Then $x \in \mathcal{A C}_{g}(I)$ and

$$
x_{g}^{\prime}(t) \in \bigcap_{i=1}^{\infty} \overline{\mathrm{co}} \bigcup_{k=i}^{\infty}\left\{\left(x_{k}\right)_{g}^{\prime}(t)\right\} \quad \text { for g-a.a. } t \in J .
$$

Proof Consider the set $\mathcal{S}=\left\{\left(x_{k}\right)_{g}^{\prime}: k \in \mathbb{N}\right\} \subset L_{g}^{1}(J)$, which is bounded thanks to condition (iii). Moreover, $\mathcal{S}$ satisfies 2 in Theorem 3.5. Indeed, let us denote by $\mathcal{L S}_{g}(J)$ the LebesgueStieltjes $\sigma$-algebra on $J$ induced by $g$ and define

$$
\nu(E)=\int_{E} L(s) d g(s), \quad E \in \mathcal{L S}_{g}(J)
$$

Then [10, Theorem 1.29] ensures that $v$ is a measure, and so given any sequence of $g$ measurable sets $\left\{E_{m}\right\}_{m \in \mathbb{N}} \subset J$ under the hypotheses of 2 in Theorem 3.5, we have

$$
\lim _{m \rightarrow \infty} \int_{E_{m}} L(s) d g(s)=\lim _{m \rightarrow \infty} v\left(E_{m}\right)=\lim _{m \rightarrow \infty} v\left(\bigcap_{k=1}^{m} E_{k}\right)=v(\emptyset)=0 .
$$

Now, condition (iii) implies that condition 2 in Theorem 3.5 holds.

Hence, $\mathcal{S}$ is weakly sequentially compact. Thus there exists a subsequence $\left\{\left(x_{k_{l}}\right)_{g}^{\prime}\right\}_{l \in \mathbb{N}}$ converging weakly to a function, say $y \in L_{g}^{1}(J)$, which implies that for every $t \in I$ we have

$$
x(t)=\lim _{l \rightarrow+\infty} x_{k_{l}}(t)=\lim _{l \rightarrow+\infty}\left(x_{k_{l}}\left(t_{0}\right)+\int_{\left[t_{0}, t\right)}\left(x_{k_{l}}\right)_{g}^{\prime}(s) d g(s)\right)=x\left(t_{0}\right)+\int_{\left[t_{0}, t\right)} y(s) d g(s) .
$$

Hence $x \in \mathcal{A C}_{g}(I)$ and $x_{g}^{\prime}(t)=y(t)$ for $g$-a.a. $t \in I$, see Theorem 2.2.

Now, Theorem 3.6 ensures the existence of a sequence of convex combinations of $\left(x_{k_{l}}\right)_{g}^{\prime}$, which we denote by $\left\{y_{j}\right\}_{j \in \mathbb{N}}$, converging strongly in $L_{g}^{1}(J)$ to $y$. Hence, there exists a subsequence $\left\{y_{j_{q}}\right\}_{q \in \mathbb{N}}$ such that $y_{j_{q}}(t) \rightarrow y(t)$ for $g$-a.a. $t \in J$. For every $q \in \mathbb{N}$ we have

$$
y_{j_{q}}(t) \in \operatorname{co} \bigcup_{l=1}^{\infty}\left\{\left(x_{k_{l}}\right)_{g}^{\prime}(t)\right\} \subset \operatorname{co} \bigcup_{k=1}^{\infty}\left\{\left(x_{k}\right)_{g}^{\prime}(t)\right\}
$$

so going to the limit as $q$ tends to infinity, we get

$$
x_{g}^{\prime}(t)=y(t) \in \overline{c o} \bigcup_{k=1}^{\infty}\left\{\left(x_{k}\right)_{g}^{\prime}(t)\right\}, \quad g \text {-a.a. } t \in I .
$$

Moreover, since for each fixed $i \in \mathbb{N}$ the sequence $\left\{x_{k}\right\}_{k=i}^{\infty}$ also converges to $x$, a repetition of the previous arguments shows that

$$
x_{g}^{\prime}(t) \in \overline{\mathrm{co}} \bigcup_{k=i}^{\infty}\left\{\left(x_{k}\right)_{g}^{\prime}(t)\right\}, \quad g \text {-a.a. } t \in I .
$$

Hence,

$$
x_{g}^{\prime}(t) \in \bigcap_{i=1}^{\infty} \overline{\operatorname{co}} \bigcup_{k=i}^{\infty}\left\{\left(x_{k}\right)_{g}^{\prime}(t)\right\}, \quad g \text {-a.a. } t \in I .
$$


We now have all the necessary tools to prove the following existence result for (3.2). To the best of the authors' knowledge, this result is new even in the particular case of $g(t)=t$, i.e., when $g$-derivatives reduce to derivatives in the usual sense.

Theorem 3.9 Let $F: I \times \mathbb{R}^{n} \rightarrow \mathcal{P}\left(\mathbb{R}^{n}\right)$ satisfy $(\mathrm{H} 1)-(\mathrm{H} 3)$. Then (3.2) has at least a solution.

Proof Consider the sequence $x_{k}:\left[t_{0}, t_{0}+T\right] \rightarrow \mathbb{R}^{n}, k \in \mathbb{N}$, defined as follows: for each $k \in \mathbb{N}$, denote $t_{k, j}=t_{0}+j T / k, j=0,1,2, \ldots, k$. Define $x_{k}\left(t_{0}\right)=x_{0}$,

$$
x_{k}(t)=x_{k}\left(t_{k, j}\right)+\int_{\left[t_{k, j}, t\right)} f_{k, j}(s) d g(s), \quad t \in\left(t_{k, j}, t_{k, j+1}\right], j=0,1,2, \ldots, k-1
$$

where $f_{k, j}$ is a $g$-measurable selection of $F\left(\cdot, x_{k}\left(t_{k, j}\right)\right), j=0,1,2, \ldots, k-1$, whose existence is guaranteed by (H2). Then, if we define $f_{k}:\left[t_{0}, t_{0}+T\right) \rightarrow \mathbb{R}^{n}$ as

$$
f_{k}(t)=f_{k, j}(t), \quad t \in\left[t_{k, j}, t_{k, j+1}\right), j=0,1,2, \ldots, k-1,
$$

it follows that

$$
x_{k}(t)=x_{0}+\int_{\left[t_{0}, t\right)} f_{k}(s) d g(s), \quad t \in\left[t_{0}, t_{0}+T\right]
$$

Moreover, Remark 3.4 ensures that $f_{k} \in L_{g}^{1}\left(\left[t_{0}, t_{0}+T\right)\right)$ and so $\left\{x_{k}\right\}_{k \in \mathbb{N}}$ is well-defined and Theorem 2.2 implies that $x_{k} \in \mathcal{A C}_{g}(I)$ and $x_{g}^{\prime}(t)=f_{k}(t)$ for $g$-a.a. $t \in J$.

Note that $\left\{x_{k}\left(t_{0}\right): k \in \mathbb{N}\right\}=\left\{x_{0}\right\}$ and for every $k \in \mathbb{N}$ we have

$$
\left\|\left(x_{k}\right)_{g}^{\prime}(t)\right\|=\left\|f_{k}(t)\right\| \leq L(t), \quad g \text {-a.a. } t \in\left[t_{0}, t_{0}+T\right),
$$

so Proposition 2.7 guarantees that $\left\{x_{k}\right\}_{k \in \mathbb{N}}$ is a relatively compact subset of $\mathcal{B C}_{g}\left(\left[t_{0}, t_{0}+T\right]\right)$. Therefore, there exists a subsequence converging to a function, say $x$. Clearly, $x\left(t_{0}\right)=x_{0}$. Moreover, applying Lemma 3.8 to such subsequence, we deduce that $x \in \mathcal{A C}_{g}(I)$ and there exists $E \subset J$ such that $\mu_{g}(E)=0$ and

$$
x_{g}^{\prime}(t) \in \bigcap_{j=1}^{\infty} \overline{\mathrm{co}} \bigcup_{k=j}^{\infty}\left\{\left(x_{k}\right)_{g}^{\prime}(t)\right\}=\bigcap_{j=1}^{\infty} \overline{\mathrm{co}} \bigcup_{k=j}^{\infty}\left\{f_{k}(t)\right\}, \quad t \in J \backslash E .
$$

Let us prove that

$$
x_{g}^{\prime}(t) \in \bigcap_{r>0}^{\infty} \overline{\operatorname{co}} F\left(t, B_{r}(x(t))\right) \quad \text { for all } t \in J \backslash E .
$$

Fix $t \in J \backslash E$; for each $k \in \mathbb{N}$, we can find $i_{k} \in\{0,1,2, \ldots, k-1\}$ such that $t \in\left[t_{k, i_{k}}, t_{k, i_{k}+1}\right)$. Hence,

$$
x_{g}^{\prime}(t) \in \bigcap_{j=1}^{\infty} \overline{\mathrm{co}} \bigcup_{k=j}^{\infty}\left\{f_{k}(t)\right\} \subset \bigcap_{j=1}^{\infty} \overline{\mathrm{co}} \bigcup_{k=j}^{\infty} F\left(t, x_{k}\left(t_{k, i_{k}}\right)\right) .
$$


Moreover, note that $t_{k, i_{k}}$ converges to $t$ from the left as $k \rightarrow \infty$, because

$$
0 \leq t-t_{k, i_{k}}<t_{k, i_{k}+1}-t_{k, i_{k}}=\frac{T}{k}
$$

Therefore, since each $x_{k}$ is left-continuous (see Proposition 2.5) and $x_{k}(t)$ converges to $x(t)$, it follows that $x_{k}\left(t_{k, i_{k}}\right)$ converges to $x(t)$. Hence, for every $r>0$ there exists $k_{0} \in \mathbb{N}$ such that

$$
\left\|x_{k}\left(t_{k, i_{k}}\right)-x(t)\right\|<r \quad \text { for all } k \geq k_{0}
$$

and therefore (3.5) yields

$$
x_{g}^{\prime}(t) \in \overline{\mathrm{co}} \bigcup_{k=k_{0}}^{\infty} F\left(t, x_{k}\left(t_{k, i_{k}}\right)\right) \subset \overline{\mathrm{co}} F\left(t, B_{r}(x(t))\right) \text {. }
$$

This implies (3.4) because $r>0$ was arbitrary.

Now we are ready for the proof of $x_{g}^{\prime}(t) \in F(t, x(t))$ for $g$-a.a. $t \in J \backslash E$. We start by removing some inconvenient $g$-null measure sets from $J \backslash E$. For each $p \in \mathbb{N}$, define the set

$$
A_{p}=\left\{t \in J \backslash\left(E \cup D_{g}\right): x(t) \in K_{p}(t), x(s) \notin K_{p}(s) \text { for all } s \in\left(t, t+\varepsilon_{t}\right) \text { and some } \varepsilon_{t}>0\right\} .
$$

For every $t \in A_{p}$ take the greatest $\varepsilon_{t}$ possible in the conditions of the definition of $A_{p}$. The infinite sum

$$
\sum_{t \in A_{p}} \varepsilon_{t}
$$

is convergent (bounded by the length of the interval $J$ ) and therefore, at most a countable set of $\varepsilon_{t}$ can be positive. Hence $\mu_{g}\left(A_{p}\right)=0$ because $A_{p}$ is countable and contains no discontinuity points of $g$.

We consider the $g$-null measure set $\hat{E}=E \cup A_{1} \cup A_{2} \cup \cdots$ and, without loss of generality, we assume that condition (H1) (ii) is satisfied for all $t \in J \backslash\left(\hat{E} \cup D_{g}\right)$.

Now we fix $t \in J \backslash \hat{E}$ and we consider two cases to prove that $x_{g}^{\prime}(t) \in F(t, x(t))$.

Case I: $t \in J \backslash\left(\hat{E} \cup D_{g}\right)$ and $x(t) \in K_{p}(t)$ for some $p \in \mathbb{N}$. Since $t \notin A_{p}$, there exist numbers

$$
h_{k}>0, h_{k} \rightarrow 0 \text { as } k \rightarrow \infty \text {, and } x\left(t+h_{k}\right) \in K_{p}\left(t+h_{k}\right) \text { for all } k,
$$

then the definition of contingent $g$-derivative ensures that

$$
x_{g}^{\prime}(t)=\lim _{k \rightarrow \infty} \frac{x\left(t+h_{k}\right)-x(t)}{g\left(t+h_{k}\right)-g(t)} \in \mathrm{D}_{g} K_{p}(t, x(t)) .
$$

Thus, condition (H1)(ii) implies that $x_{g}^{\prime}(t) \in F(t, x(t))$.

Case II. $t \in J \cap D_{g}$ or $t \in J \backslash\left(\hat{E} \cup D_{g}\right)$ and $x(t) \notin K(t)$. In both cases, condition (H1) ensures that $F(t, x(t))$ is convex and compact and $F(t, \cdot)$ is usc at $x(t)$.

Reasoning by contradiction, assume that $x_{g}^{\prime}(t) \notin F(t, x(t))$. Then, since $F(t, x(t))$ is a compact subset of $\mathbb{R}^{n}$, Lemma 3.7 implies that there exists $\varepsilon_{0}>0$ such that

$$
x_{g}^{\prime}(t) \notin F(t, x(t))+B_{\varepsilon_{0}}(0) .
$$


Moreover, since $F(t, \cdot)$ is usc, it is $\varepsilon$ - $\delta$-usc (see [5, Proposition 1.1]), so there exists $\delta>0$ such that

$$
F(t, y) \subset F(t, x(t))+B_{\varepsilon_{0} / 2}(0), \quad \text { for all } y \in \mathbb{R}^{n},\|y-x(t)\|<\delta .
$$

Take $k_{0} \in \mathbb{N}$ such that

$$
\left\|x_{k}\left(t_{k, i_{k}}\right)-x(t)\right\|<\delta \quad \text { for all } k \geq k_{0} .
$$

Then (3.8) yields

$$
F\left(t, x_{k}\left(t_{k, i_{k}}\right)\right) \subset F(t, x(t))+B_{\varepsilon_{0} / 2}(0) \subset F(t, x(t))+\overline{B_{\varepsilon_{0} / 2}(0)} \quad \text { for all } k \geq k_{0} .
$$

Now, since $F(t, x(t))+\overline{B_{\varepsilon_{0} / 2}(0)}$ is a convex and closed set, it follows that

$$
\overline{\mathrm{co}} \bigcup_{k=k_{0}}^{\infty} F\left(t, x_{k}\left(t_{k, i_{k}}\right)\right) \subset F(t, x(t))+\overline{B_{\varepsilon_{0} / 2}(0)} \subset F(t, x(t))+B_{\varepsilon_{0}}(0),
$$

and we deduce from (3.5) that $x_{g}^{\prime}(t) \in F(t, x(t))+B_{\varepsilon_{0}}(0)$, a contradiction with (3.7).

The result of Theorem 3.9 may fail if (3.3) is not satisfied just at one point as we show in the following example.

Example 3.10 Problem (3.2) with $g(t)=t, t_{0}=0, T=1, x_{0}=0$, and

$$
F(t, x)= \begin{cases}\{1\} & \text { if } x<0 \\ \{1 / 2\} & \text { if } x=0 \\ \{-1\} & \text { if } x>0\end{cases}
$$

has no solution. Observe that $F(t, \cdot)$ assumes convex and compact values and it is usc on $\mathbb{R} \backslash\{0\}$.

In this case we should take $K(t)=\{0\}$ for all $t \in[0,1]$, and we have

$$
\bigcap_{r>0} \overline{\mathrm{co}} F(t,(-r, r)) \cap \mathrm{D} K(t, 0)=[-1,1] \cap\{0\}=\{0\} \not \subset F(t, 0) .
$$

Our next example shows that Theorem 3.9 is so general that it can be applied in cases where the nonlinear part is not usc or convex and compact valued on dense subsets of $\mathbb{R}^{n}$. Once again we consider the particular case of $g(t)=t$ to highlight that Theorem 3.9 is new even in the classical setting of usual derivatives.

Example 3.11 We shall construct an ill-behaved multivalued mapping $F:[0,1] \times \mathbb{R} \longrightarrow$ $\mathcal{P}(\mathbb{R})$ using a function $\varphi: \mathbb{R} \rightarrow \mathbb{R}$ which is discontinuous at every rational number.

First, consider a bijection $r: \mathbb{N} \longrightarrow \mathbb{Q}$, denote $r_{p}=r(p)$ for each $p \in \mathbb{N}$, and define

$$
\varphi(x)=\sum_{r_{p}<x} 2^{-p} \quad \text { for each } x \in \mathbb{R} .
$$


Obviously, $0<\varphi(x)<1$ for all $x \in \mathbb{R}$ and $\varphi$ is increasing. Moreover, $\varphi$ is continuous at every irrational and discontinuous at every rational. More precisely, for each $p \in \mathbb{N}$ we have

$$
\varphi\left(r_{p}^{-}\right)=\varphi\left(r_{p}\right)<\varphi\left(r_{p}\right)+2^{-p}=\varphi\left(r_{p}^{+}\right) .
$$

This implies that we can find $\delta_{p}>0$ such that

$$
\left|y-r_{p}\right|<\delta_{p} \quad \Rightarrow \quad \varphi(y)>\varphi\left(r_{p}\right) / 2 .
$$

Now we fix $\lambda \in(0,1)$ and we define $F(t, x)$ for a.a. $t \in I=[0,1]$ and all $x \in \mathbb{R}$ as follows:

$$
F(t, x)= \begin{cases}{[\lambda \varphi(x), \varphi(x)]} & \text { for } t \in A, x \notin \mathbb{Q}, \\ (\lambda \varphi(x), \varphi(x)) \cap \mathbb{Q} & \text { for } t \in A, x \in \mathbb{Q}, \\ {[-\varphi(x-t),-\lambda \varphi(x-t)]} & \text { for } t \in B, x-t \notin \mathbb{Q}, \\ (-\varphi(x-t),-\lambda \varphi(x-t)) \cap \mathbb{Q} & \text { for } t \in B, x-t \in \mathbb{Q},\end{cases}
$$

where

$$
A=\bigcup_{l=1}^{\infty}\left(\frac{1}{2 l}, \frac{1}{2 l-1}\right) \text { and } B=\bigcup_{l=1}^{\infty}\left(\frac{1}{2 l+1}, \frac{1}{2 l}\right) .
$$

Note that $F$ is not explicitly defined for $t \in C=\left\{(2 l)^{-1}: l \in \mathbb{N}\right\} \cup\{0\}$, nor is it necessary as it is countable, and thus $m(C)=0$. We shall show that the hypotheses of Theorem 3.9 are satisfied regardless of the values of $F$ on $C \times \mathbb{R}$. Also note that $F(t, x)$ is neither convex nor compact if $t \in A$ and $x \in \mathbb{Q}$ or if $t \in B$ and $x-t \in \mathbb{Q}$. Moreover, if $t \in A$, then $F(t, \cdot)$ is not usc at rational numbers because $\varphi$ jumps upwards at rationals.

Clearly, (H3) is satisfied with $M(t)=1, t \in[0,1]$. Condition (H2) is easy to check: for each fixed $x \in \mathbb{R} \backslash \mathbb{Q}$, we can take the selection $f_{x}(t)=\varphi(x)$ for $t \in A$, and $f_{x}(t)=-\varphi(x-t)$ for $t \in B$. Observe that $f_{x}(t)$ may not be an element of $F(t, x)$ on $C_{x}=\{t \in B: x-t \in \mathbb{Q}\}=B \cap(x-\mathbb{Q})$, but this does not matter because $C_{x}$ is a countable set. For the case $x \in \mathbb{Q}$, just take any $q \in(\lambda \varphi(x), \varphi(x)) \cap \mathbb{Q}$ and consider $f_{x}(t)=q$ for $t \in A, f_{x}(t)=-\varphi(x-t)$ for $t \in B$. In any case, $f_{x}$ is piecewise monotone, hence measurable.

Now for condition (H1). Define

$$
K(t)=\bigcup_{p=1}^{\infty} K_{p}(t), \quad t \in A \cup B
$$

where, for each $p \in \mathbb{N}$,

$$
K_{p}(t)= \begin{cases}\left\{r_{p}\right\} & \text { if } t \in A \\ \left\{t+r_{p}\right\} & \text { if } t \in B\end{cases}
$$

Clearly, $\mathrm{D} K_{p}\left(t, r_{p}\right)=\{0\}$ for all $t \in A$, and

$$
\mathrm{D} K_{p}\left(t, t+r_{p}\right)=\{1\} \quad \text { for all } t \in B
$$


For a.a. $t \in I$ and every $x \in \mathbb{R} \backslash K(t)$, the set $F(t, x)$ is closed and convex.

Let us prove that, for a.a. $t \in I$, the multivalued mapping $F(t, \cdot)$ is usc at every fixed $x \in \mathbb{R} \backslash K(t)$. If $t \in A$, then $\varphi$ is continuous at $x$, so for each $\varepsilon>0$ there exists $\delta>0$ such that

$$
|y-x|<\delta \Rightarrow|\varphi(y)-\varphi(x)|<\varepsilon \Rightarrow|\mu \varphi(y)-\mu \varphi(x)|<\varepsilon \quad \text { for any } \mu \in[-1,1] \text {. }
$$

Hence, if $t \in A$, and $|y-x|<\delta$, then

$$
F(t, y) \subset[\lambda \varphi(y), \varphi(y)] \subset(\lambda \varphi(x)-\varepsilon, \varphi(x)+\varepsilon)=F(t, x)+(-\varepsilon, \varepsilon) .
$$

The proof is similar for $t \in B$, but the only difference is that we have to use the fact that $\varphi$ is continuous at $x-t$, because $x \notin K_{p}(t)=\left\{t+r_{p}\right\}$ for any $p$.

Finally, we have to check that (3.3) holds. If $t \in A$, then, for each fixed $p \in \mathbb{N}$, there exists $\delta_{p}>0$ such that (3.9) holds if $\left|y-r_{p}\right|<\delta_{p}$. Then, if $\left|y-r_{p}\right|<\delta_{p}$ and $z \in F(t, y) \subset[\lambda \varphi(y), \varphi(y)]$, then

$$
z \geq \lambda \varphi(y)>\lambda \varphi\left(r_{p}\right) / 2
$$

Hence

$$
\overline{\operatorname{co}} F\left(t,\left(r_{p}-\delta_{p}, r_{p}+\delta_{p}\right)\right) \subset\left[\lambda \varphi\left(r_{p}\right) / 2, \infty\right)
$$

which implies that

$$
\bigcap_{r>0} \overline{\operatorname{co}} F\left(t,\left(r_{p}-r, r_{p}+r\right)\right) \subset\left[\lambda \varphi\left(r_{p}\right) / 2, \infty\right) \subset(0, \infty) .
$$

Then the intersection in (3.3) is empty and therefore condition (3.3) holds.

Checking condition (3.3) for $t \in B$ and $x=t+r_{p}$ for some $p$ is easier. Clearly,

$$
\bigcap_{r>0} \overline{\mathrm{Co}} F\left(t,\left(t+r_{p}-r, t+r_{p}+r\right)\right) \subset(-\infty, 0]
$$

and $D K_{p}\left(t, t+r_{p}\right)=\{1\}$.

\section{An existence result for Stieltjes differential equations}

Let the intervals $I$ and $J$ be as in (3.1) and consider the following initial value problem:

$$
x_{g}^{\prime}(t)=f(t, x(t)), \quad g \text {-a.a. } t \in J, \quad x\left(t_{0}\right)=x_{0} .
$$

As a corollary of Theorem 3.9 we obtain the following existence principle for (4.1).

Theorem 4.1 Let $f: J \times \mathbb{R}^{n} \rightarrow \mathbb{R}^{n}$ satisfy (i), (ii) and

(i) for all $x \in \mathbb{R}^{n}$, the map $f(\cdot, x)$ is g-measurable;

(ii) there exists $L \in L_{g}^{1}(J,[0, \infty))$ and $N \subset J$ with $\mu_{g}(N)=0$, such that

$$
\|f(t, x)\| \leq L(t), \quad \text { for all } x \in \mathbb{R}^{n} \text { and all } t \in J \backslash N \text {; }
$$


(iii) for all $t \in J \backslash\left(N \cup D_{g}\right), f(t, \cdot)$ is continuous on $\mathbb{R}^{n} \backslash K(t)$, where $K(t)=\bigcup_{p=1}^{\infty} K_{p}(t)$, and for each $p \in \mathbb{N}$ and $x \in K_{p}(t)$, we have

$$
\bigcap_{r>0}^{\infty} \overline{\operatorname{co}} f\left(t, B_{r}(x)\right) \cap \mathrm{D}_{g} K_{p}(t, x) \subset\{f(t, x)\}
$$

(iv) for all $t \in D_{g}, f(t, \cdot)$ is continuous on $\mathbb{R}^{n}$.

Then the initial value problem (4.1) has at least one solution.

To conclude this section, we present the following example in which is possible to apply the previous result to obtain the existence of solution for the corresponding initial value problem.

Example 4.2 Let $g: \mathbb{R} \rightarrow \mathbb{R}$ be a nondecreasing left-continuous map, $\varphi$ as in Example 3.11 and $\psi$ a continuous function such that $\psi^{-1}(\mathbb{Q})$ is countable. Define $f: J \times \mathbb{R} \rightarrow \mathbb{R}$ as

$$
f(t, x)=\varphi\left(\psi(\alpha x+\beta g(t)) \chi_{J \backslash D_{g}}(t)+t \chi_{D_{g}}(t)\right),
$$

with $\alpha>0, \beta \in(-\infty,-\alpha) \cup(0,+\infty)$. Note that $0<f(t, x)<1$ for all $(t, x) \in J \times \mathbb{R}$, so

$$
\bigcap_{r>0}^{\infty} \overline{\operatorname{co}} f\left(t, B_{r}(x)\right) \subset[0,1]
$$

for all $(t, x) \in J \times \mathbb{R}$. Consider the initial value problem

$$
x_{g}^{\prime}(t)=f(t, x), \quad t \in J, \quad x\left(t_{0}\right)=x_{0}
$$

Let us show that the previous problem has at least a solution by proving that the hypotheses of Theorem 4.1 are satisfied.

Conditions (ii) and (iv) follow directly from the definition of $f$. For condition (i), fix $x \in \mathbb{R}$. The map

$$
t \in J \mapsto \varphi\left(\psi(\alpha x+\beta g(t)) \chi_{\supset \backslash D_{g}}(t)+t \chi_{D_{g}}(t)\right)
$$

is Borel-measurable as it is the composition of Borel-measurable maps. Hence, it is $g$ measurable as the Borel $\sigma$-algebra is contained in the Lebesgue-Stieltjes $\sigma$-algebra defined by $g$.

Finally, for condition (iii), write $\psi^{-1}(\mathbb{Q})=\left\{s_{p}: p \in \mathbb{N}\right\}$ and define $\gamma_{p}: J \rightarrow \mathbb{R}, p \in \mathbb{N}$, as

$$
\gamma_{p}(t)=\frac{s_{p}-\beta g(t)}{\alpha}
$$

$K_{p}: J \rightarrow \mathcal{P}(\mathbb{R}), p \in \mathbb{N}$, as $K_{p}(t)=\left\{\gamma_{p}(t)\right\}$ and $K(t)=\bigcup_{p=1}^{\infty} K_{p}(t)$. Note that, for each $p \in \mathbb{N}$, $\gamma_{p}$ is $g$-differentiable everywhere in $J$ and $\gamma_{g}^{\prime}(t)=-\beta / \alpha, t \in J$. Therefore, $\mathrm{D}_{g} K_{p}\left(t, \gamma_{p}(t)\right)=$ $\{-\beta / \alpha\}, p \in \mathbb{N}$, so $\mathrm{D}_{g} K_{p}\left(t, \gamma_{p}(t)\right) \subset(-\infty, 0) \cup(1,+\infty)$, depending on the value of $\beta$.

Fix $t \in J \backslash D_{g}$. If $x \in K_{p}(t)$ for some $p \in \mathbb{N}$, then the intersection in (4.2) is empty, so the condition is trivially satisfied. On the other hand, if $x \in \mathbb{R} \backslash K(t)$, it follows that $\psi(\alpha x+$ 
$\beta g(t)) \notin \mathbb{Q}$ since

$$
\alpha x+\beta g(t) \neq s_{p}, \quad \text { for all } p \in \mathbb{N} .
$$

Hence $\varphi$ is continuous at $\psi(\alpha x+\beta g(t))$ and so $f(t, \cdot)$ is continuous at $x$ as it is the composition of continuous functions. That is, the hypotheses of Theorem 4.1 are satisfied and so the initial value problem has at least a solution.

\section{Acknowledgements}

The authors would like to thank the anonymous referees for their encouraging reports and comments, which helped to improve this manuscript.

\section{Funding}

Rodrigo López Pouso was partially supported by Ministerio de Economía y Competitividad, Spain, and FEDER, Project MTM2016-75140-P and Xunta de Galicia under grant ED431C 2019/02. Ignacio Márquez Albés was partially supported by Ministerio de Economía y Competitividad, Spain, and FEDER, Project MTM2016-75140-P and Xunta de Galicia under grants ED481A-2017/095 and ED431C 2019/02. Jorge Rodríguez-López was partially supported by Ministerio de Economía y Competitividad, Spain, and FEDER, Project MTM2016-75140-P and Xunta de Galicia under grants ED481A-2017/178 and ED431C 2019/02.

\section{Availability of data and materials}

Not applicable.

\section{Competing interests}

The authors declare that they have no competing interests.

\section{Authors' contributions}

All authors contributed equally to the different parts of the manuscript. All authors read and approved the final manuscript.

\section{Publisher's Note}

Springer Nature remains neutral with regard to jurisdictional claims in published maps and institutional affiliations.

Received: 10 January 2020 Accepted: 6 May 2020 Published online: 20 May 2020

\section{References}

1. Aubin, J.P., Cellina, A.: Differential Inclusions. Springer, Berlin (1984)

2. Cid, J.Á., López Pouso, R.: Ordinary differential equations and systems with time-dependent discontinuity sets. Proc. R. Soc. Edinb., Sect. A 134(4), 617-637 (2004)

3. Daniell, P.J.: Differentiation with respect to a function of limited variation. Trans. Am. Math. Soc. 19(4), 353-362 (1918)

4. Davy, J.L.: Properties of the solution set of a generalized differential equation. Bull. Aust. Math. Soc. 6, 379-398 (1972)

5. Deimling, K.: Multivalued Differential Equations. de Gruyter, Berlin (1992)

6. Dunford, N., Schwartz, J.T.: Linear Operators, Part I. Wiley, New York (1958)

7. Frigon, M., López Pouso, R.: Theory and applications of first-order systems of Stieltjes differential equations. Adv. Nonlinear Anal. 6(1), 13-36 (2017)

8. López Pouso, R., Rodríguez, A.: A new unification of continuous, discrete, and impulsive calculus through Stieltjes derivatives. Real Anal. Exch. 40(2), 319-354 (2015)

9. Monteiro, G.A., Satco, B.: Extremal solutions for measure differential inclusions via Stieltjes derivatives. Adv. Differ. Equ. 2019, 239 (2019)

10. Rudin, W.: Real and Complex Analysis. McGraw-Hill, New York (1987) 